\title{
Implantable microcoil for in-vivo magnetic resonance spectroscopy
}

\begin{abstract}
Nuclear Magnetic Resonance (NMR) allows a non-destructive study of molecular structures by the measurement of their atomic nuclei magnetization. Nonetheless, NMR technique still suffers from the sensitivity weakness compared to all other spectroscopy approaches. Therefore, the optimization of detection Radio Frequency (RF) coils and the reduction of the thermal noise will lead to an increase in signal resolution particularly around the regions of interest (ROI) within the living sample. In the case of sample with limited mass and volume, the sensitivity may be improved for when the size of the RF coil matches the sample size. In this study, we developed a microcoil $(500 \times 1000 \mu \mathrm{m})$ dedicated to the NMR micro imaging (MRI) and micro localized spectroscopy (MRS) for biomedical applications on small rodent models of human diseases. Our NMR microcoil offer a sensitivity increased by 2.5 , and limits of detection (LOD) of the main cerebral metabolites estimated by MRS invitro measurements in good concordance with analytical values given in literature. The realization of compact sensing system based on microcoil will open the door to numerous medical and industrial.
\end{abstract}

Volume 3 Issue 2 - 2017

Latifa Fakri-Bouchet, Mohammed Zahraoui
University Claude Bernard-Lyon I, France

Correspondence: Latifa Fakri-Bouchet, University of Lyon, University Claude Bernard-Lyon I, UMR CNRS 5280, Institute Des Sciences Analytiques (ISA), 5 Rue De La Doua,Villeurbanne, France, Tel 06031842176, Email latifa.bouchet@univ-lyonl.fr

Received: July 31, 2017 | Published: September 15, 2017

Keywords: nuclear magnetic resonance, spectroscopy, in-vivo, region of interest, limit of detection, radiofrequency, microcoil

Abreviations: NMR, nuclear magnetic resonance; MRS, micro localized spectroscopy; MRI, micro imaging; ROI, regions of interest; LOD, limits of detection; RF, radio frequency; $\mu$ coil, microcoil

\section{Introduction}

The Nuclear Magnetic Resonance Spectroscopy (MRS) is the study metabolic processes in different biological and chemical samples as tissues, cell cultures, and protein structures. MRS fulfils an important role through its ability to deduce structural information and to withdraw microscopic data from the intermolecular dynamics. The NMR Approach has expand its capacities to cover furthermost medical and chemical diagnosis applications. However, NMR sensitivity still lags significantly behind most other analytical techniques by a factor of 100-1000, mainly for mass-limited and concentrationlimited samples. ${ }^{1}$ The observation of metabolic processes using short spin-spin relaxation decay becomes possible via short echo-time localization pulse-sequences. Yet, spectra quantification is obstructed by overlapping metabolite resonances and by low Signal-to-Noise Ratio (SNR) due to the limited size of the observed volume. Hence, the analysis of sample volumes of nanoliter order point toward the improvement of Radio-Frequency (RF) coils having dimensions of several micrometers. ${ }^{2,3}$ The sensitivity of an NMR receiver coil is expressed as a ratio of the SNR and the concentration of the measured substance. ${ }^{4}$

$$
\text { Sensitivity }=\frac{S N R}{C_{i}}
$$

Where $\mathrm{C}_{\mathrm{i}}$ is the concentration of the measured substance in $\mathrm{mol} / \mathrm{kg}$. The SNR is the fundamental quantifier of microcoil's sensitivity due to the existence of the Noise as a limiting factor. SNR is an empirical relation transcribed as:

$$
\text { SNR }=\frac{\text { Signal Amplitude }(\text { FID })}{\text { Quadratic mean ofthe Noise }}
$$

The SNR could also be depicted analytically by the physical parameters of the microcoil and the surrounding setting:

$$
S N R \propto \omega_{0} B_{0} V_{S} \frac{B_{1} / i}{\sqrt{4 K_{B} T_{S} R_{S} \Delta f}}
$$

Where $k_{B}$ is the constant of Boltzmann, $T_{S}$ is the ambient temperature, $\mathrm{R}_{\mathrm{s}}$ is the coil conductors equivalent resistance, and $\ddot{A} f$ is the receiver bandwidth. Two main components could be differentiated: (1) The environment components are the static magnetic field $\mathrm{B}_{0}$, the Larmor precession frequency $\omega_{0}$ proportional to $\mathrm{B}_{0}$ and the sample volume $\mathrm{V}_{\mathrm{s}}$. (2) The geometrical components are $\mathrm{B}_{1} / i$ which is equivalent to the NMR signal by the principle of reciprocity and $4 K_{B} T_{S} R_{S} \Delta f$ symbolizes the thermal noise produced by the coil in a given bandwidth. ${ }^{5}$

\section{Discussion}

The experimental equipment providing the high static field $\mathrm{B}_{0}$ had are stirring toward $20 \mathrm{~T}$ magnetic resonance intended for studying human brain still they rarely available for typical research. ${ }^{6}$ Thus, the geometrical parameters are optimized to attain a maximum $\mathrm{B}_{1}$ and to reduce the coil dimensions (minimum Rs) so a low noise Signal could be detected.

\section{Microcoil geometry effect on sensitivity}

The design of a planar sub-milimetric coil recommends defining firmly the dimension so the detection size of the Radio Frequency coil 
matches the sample size. Spiral configurations, as shown in Figure 1, could be the finest solution for in-vivo implant examinations. ${ }^{7}$ From the optimization results, the square coil $\left(1000 \times 1000 \mu^{2}\right)$ have displayed a high sensitivity for the observation of tiny sample of $1000 \mu \mathrm{l}$. Although, the race track shape have numerous physical advantage such as the round corners, which reduce the effect of hotspots that can burn the sample, and especially the half surface area compared to the square coil, the thing that will reduce lesion size after the implantation inside the brain. Table 1 represents a complete comparison between geometric shapes of the microcoil considering the ratio called quality factor $\mathrm{Q}$ of the receiving coil (Figure 2):

$$
Q=\frac{L \omega}{R_{s}}
$$

Where $R_{s}$ the equivalent resistance and the admittance Los , and $\mathrm{R}_{\mathrm{AC}}$ is the $\mu$ coil Alternative Current resistance. All Magnetic Resonance Imaging (MRI) and Magnetic Resonance Spectroscopy (MRS) experiments were performed using a 4.7T-Bruker Biospec System, the resonance frequency is about $\mathrm{f}_{0}=200 \mathrm{MHz}$.

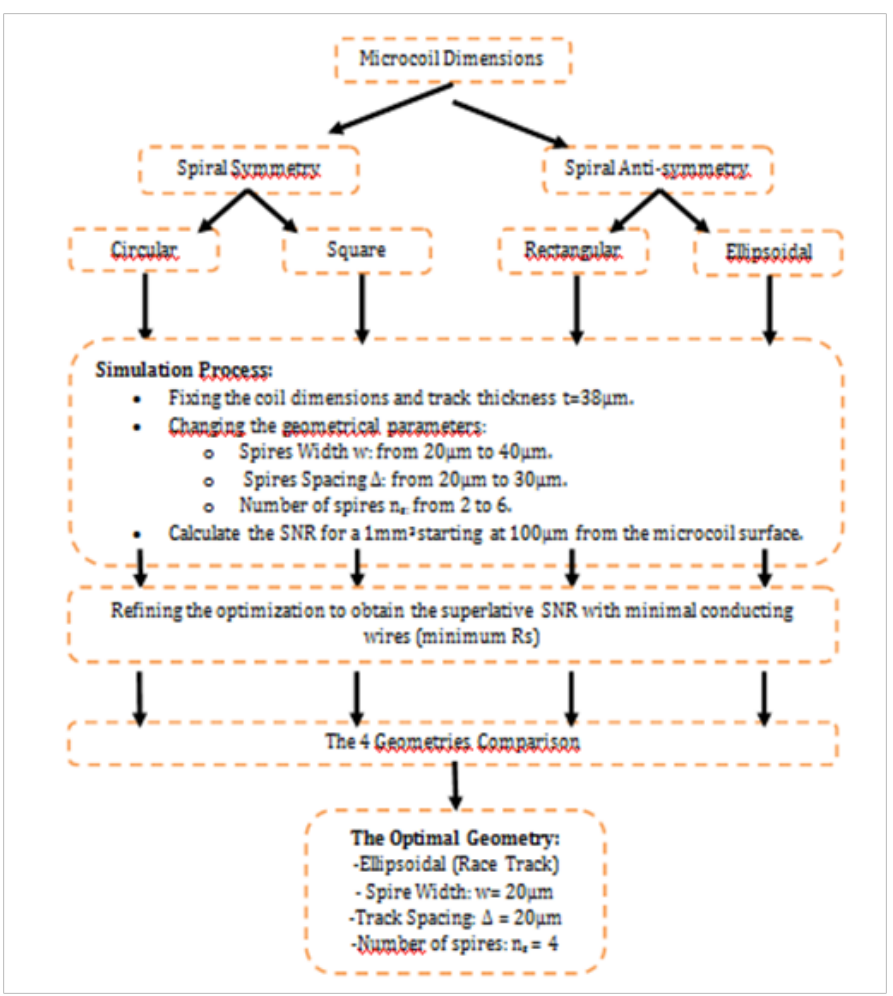

Figure I Optimization organogram of the microcoil different geometry.

\section{In-Vitro study \& the comparison with commercial surface coil}

The microcoil performance was compared to a Rapid-Biomed surface-coil (3.5cm diameter) designed for ${ }^{1} \mathrm{H}$ of rat brain. For proton metabolites detection, we have evaluated their sensitivities and limits of detection (LOD) as defined in $^{4}$ and determined the factor of gain (FOG) of the microcoil approach defined as

$$
\text { FOG }=\text { LODsurfacecoil / LOD microcoil }
$$

MRS measurements were set on a phantom containing ten cerebral metabolites solutions. The chosen planar homemade micro-coil had ellipsoidal geometry $\left(1000 \times 500 \mu^{2}\right)$, had four concentric turns, $(22 \mu \mathrm{m}$ trace width, $46 \mu \mathrm{m}$ trace thickness and $20 \mu \mathrm{m}$ spacing between turns, quality coefficient $=24$ ). The planar micro-coil was immersed in the phantom. Both coils were used for signal detection only and RF transmission was accomplished using a Rapid-Biomed birdcage coil $(6.9 \mathrm{~cm}$ diameter $)$ in active decoupling mode. Spectra were obtained using PRESS sequence $(T R / T E=5000 / 20 \mathrm{~ms}$, excited voxel size $1.5 \times 1.5 \times 1.5 \mathrm{~mm}^{3}$, depth of $0.9 \mathrm{~mm}, 256$ accumulations, experiment duration 20mn). Observations and comparisons were focused on Choline (Cho), Nacetyl-aspartate (NAA), lactate doublet (Lac) and Creatine $(\mathrm{Cr})$ with concentrations of 25,50 and $100 \mathrm{mM}$ in buffered aqueous solutions. Knowing that these cerebral metabolites are not existing biologically with high concentration inside the brain compared to the buffered aqueous solutions. According to the literature review, the concentrations are ranging in the interval of $[0.9,2.5 \mathrm{mmol} / \mathrm{kg}]$ for Cho, $[7,16 \mathrm{mmol} / \mathrm{kg}]$ for NAA and $[5.01,10.6 \mathrm{mmol} / \mathrm{kg}]$ for $\mathrm{Cr}^{8-11}$ The Figure 1 shows a spectrum obtained using micro-coil. All our Spectra were quantified using Bruker Top spin and via jMRUI software and AMARES method ${ }^{12}$ (Figure 3). LODs determined in all cases from signal-to-noise measurements were compared according to both of coil strategies and statistically processed. FOG values are reported in Figure 4 for Cho, NAA, Lac and Cr using different concentration 25, 50 and $100 \mathrm{mM}$. They show that the micro-coil approach can provide a superlative sensitivity and a lower LOD than the surface-coil. Considering significant metabolites as Cho, NAA, Lac and Cr. The FOG is between 2 and 2.4. of the gain for $\mathrm{Cr}$, Cho, NAA and Lac with concentration 25(white), 50(grey), 100(black) mM. In the proposed experiments, the two kinds of coils were used with identical electrical configurations (tuning, matching, preamp bounding). Consequently, the values of sensitivity, LOD and FOG show the advantage of the micro-coil in spite of its smaller sensitive volume to detect and to analyze a small metabolites concentration.

\section{In vivo animal NMR studies using implantable micro-coil and brain tissue response to chronically implantation}

The possibility of using a new generation of micro-coils was brought down in a recent study. It has demonstrated a prosperous potential of enhancement in terms of SNR, spatial resolution, and limits of detection (LOD), compared to the surface-coil. The originality of the present work is to show how to realize brain implantable NMR probehead, tolerated by rats for several weeks. To the best of our knowledge, no in vivo study has been already established with such sensing methodology. Reinforced by acquisition and signal processing, the aim is to push to the limits of in vivo detection and an ultra-localization technique should result from this approach. For in vivo implantation, we have conducted the experiment on rats Wistar (230-300g). The animals handling was carried out according to recommendations of the local ethics committee. ${ }^{13}$ The micro-coil, "needle" part only, was introduced into the brain by stereotaxy (DavidKopf). First results demonstrate that micro-coil positioning in intracerebral structure is well reproducible under stereotaxic conditions. With chronic implantation, the rats survived up to 4 months. Consequently, the biocompatibility aspect is also validated. Axial MRI slices, Figure 5A, allow measurements of the lesion dimensions, compared then with the micro-coil size and to stereotaxic coordinates determined thanks to the rat atlas ${ }^{14}$ and histological control, Figure 5B. The chosen location coordinates (artery basilar, cerebellum) are visible by MRI. The obtained in vivo water proton spectrum opens cerebral metabolites measurements capabilities as shown in Figure 6. 
Table I Configurations study of the different geometries with the optimal parameters

\begin{tabular}{llllllll}
\hline Configuration & $\mathbf{n}_{\mathrm{s}}($ Spires) & $\mathbf{W}(\boldsymbol{\mu m})$ & $\Delta(\boldsymbol{\mu m})$ & $\mathbf{R}_{\mathbf{A C}}(\Omega)$ & $\mathbf{L}(\mathbf{n H})$ & $\mathbf{Q}$ & $\mathbf{B}_{I_{\max }}$ \\
\hline Square & 6 & 29 & 20 & 0.98 & 33.2 & 42.5 & 1.53 \\
Circular & 6 & 29 & 20 & 0.82 & 28.2 & 43.3 & 1.31 \\
Rectangular & 4 & 22 & 20 & 0.64 & 12.7 & 25.1 & 0.62 \\
Ellipsoidal & 4 & 22 & 20 & 0.59 & 12.3 & 26.3 & 0.59 \\
\hline
\end{tabular}

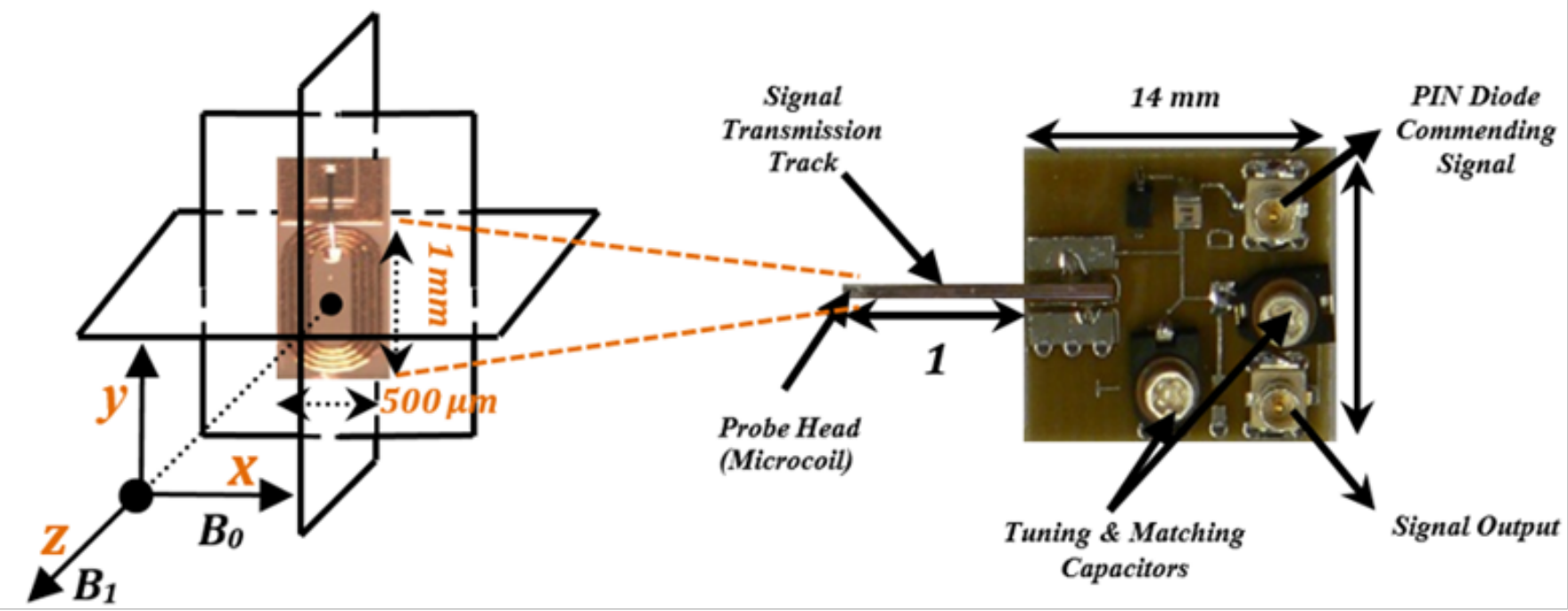

Figure 2 Microcoil with a focus on the active part with the three directions slice.

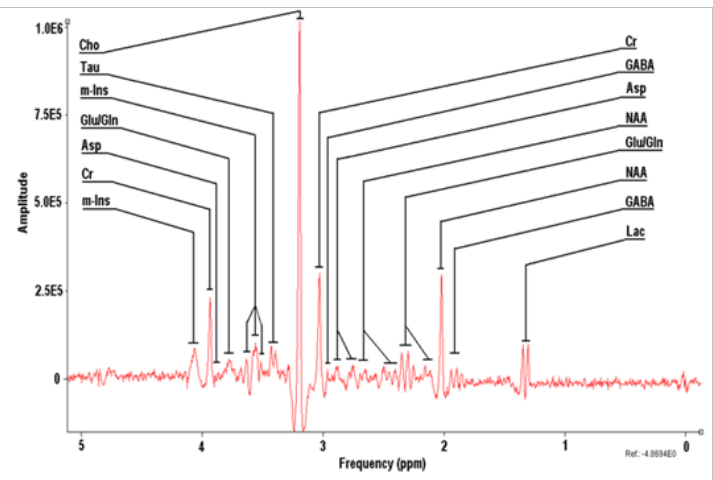

Figure 3 Spectrum of 10 metabolites for concentrations of $50 \mathrm{mM}$.

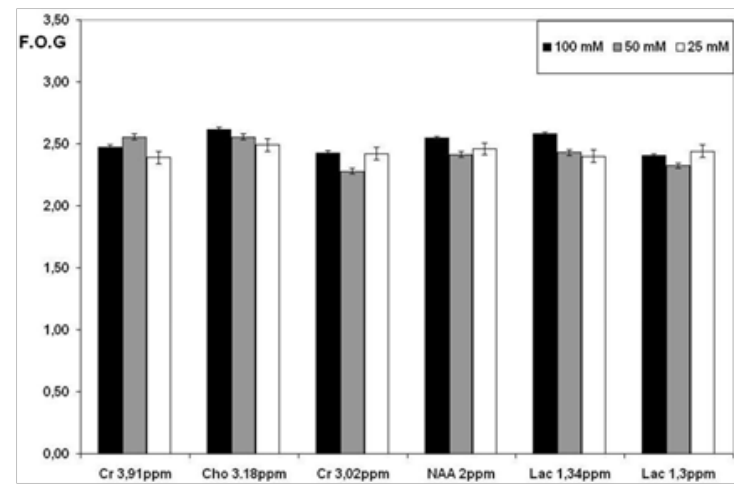

Figure 4 The factor of the gain for $\mathrm{Cr}$, Cho, NAA and Lac with concentration 25(white), 50(grey), I00(black) mM.

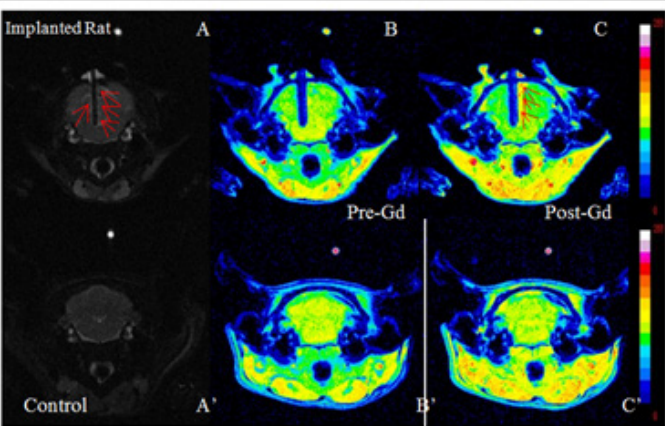

(a)

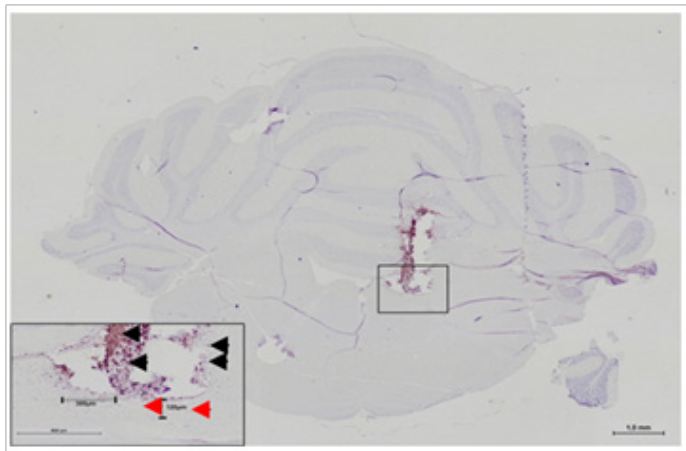

(b)

Figure 5

5a: MRI axial slice.

5b: histological slice $(40 \mu \mathrm{m})$ 


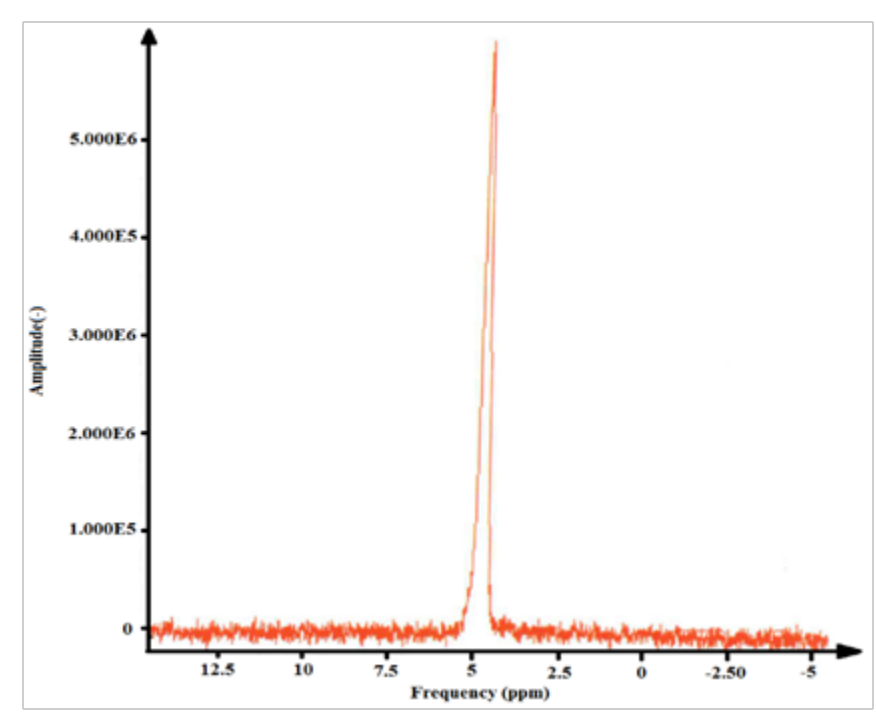

Figure 6 In vivo water Proton spectrum acquired with an implanted microcoil in a rat brain.

\section{Conclusion}

An ultra-localization technique will result from the development of this planar microcoil, due to its high spatial resolution primarily when focusing on a specific ROI. Comparing to the surface coil, the planar microcoil have shown a lower limits of detection (LOD) by an average FOG of 2.5 and higher SNR despite its small sensitive volume of detection. The LOD estimated by MRS measurements are in good concordance with analytical values given in literature. These preliminary in-vitro results enabled us to open largely the biomedical applications based on cerebral metabolism investigation by experiments in small animals. Subsequently the proposed "needlecoil" concept implies a breakthrough in biomedical research towards NMR micro-detector used for brain exploration for early diagnosis and treatment follow up. Future work will focus on the design of a Low-noise Conditioning system to amplify furthermore the NMR Signal in order to detect lower LOD of cerebral metabolites. The microcoil approach is a newborn tool that could be exploited in several biomedical applications as Alzheimer's disease \& Brain Tumors Diagnosis.

\section{Acknowledgements}

I really want to thank all my PhD students (N. Baxan (2008), A. Kadjo (2011), T. Cong-Truong (2014), J. Trejo-Rosillo (2014), who contributed through theirs thesis to this project advancement.

\section{Conflict of interest}

The author declares no conflict of interest.

\section{References}

1. Baxan N, Rabeson H, Pasquet G, et al. Limit of detection of cerebral metabolites by localized NMR spectroscopy using microcoils. Comptes Rendus Chimie. 2008;11(4-5):448-456.

2. Schoeniger JS, Aiken N, Hsu E, et al. Relaxation-time and diffusion NMR microscopy of single neurons. J Magn Reson B. 1994;103(3):261-273.

3. Peck TL, Magin RL, Lauterbur PC. Design and analysis of microcoils for NMR microscopy. J Magn Reson B. 1995;108(2):114-124.

4. Lacey ME, Subramanian R, Olson DL, et al. High-resolution NMR spectroscopy of sample volumes from $1 \mathrm{~nL}$ to $10 \mathrm{Ml}$. Chemical Reviews. 1999;99(10):3133-3152.

5. Nyquist H. Thermal agitation of electric charge in conductors. Physical Review. 1928;32(1):110-113.

6. Budinger TF, Bird MD, Frydman L, et al. Toward $20 \mathrm{~T}$, magnetic resonance for human brain studies: opportunities for discovery and neuroscience rationale. Magnetic Resonance Materials in Physics, Biology and Medicine. 2016;29(3):617-639.

7. Cong Truong T. Optimisation par approche physique des micro-antennes RMN fabriquées par Techniques Microélectroniques: Étude Théorique et Expérimentale. Thesis. 2014;3:101-105.

8. Graaf RA. In vivo NMR spectroscopy: Principles and techniques. 2nd ed. USA: John Wiley, Sons Ltd; 1998. 592 p.

9. Pfeuffer J, Tkác I, Provencher SW, et al. Toward an In Vivo Neurochemical Profile: Quantification of 18 Metabolites in Short Echo-Time 1H Spectra of the rat Brain. J Magn Reson. 1999;141(1):104-120.

10. Govindaraju V, Young K, Maudsley AA. Proton NMR chemical shifts and coupling constants for brain metabolites. NMR Biomed. 2000;3(3):129153.

11. Tkác I, Rao R, Georgieff MK, et al. Developmental and regional changes in the neurochemical profile of the rat brain determined by In Vivo $1 \mathrm{H}$ NMR spectroscopy. Magn Reson Med. 2003;50(1):24-32.

12. Kadjo A, Baxan N, Briguet A, et al. In vivo animal NMR studies using implantable microcoil. IEEE international workshop on imaging systems and techniques - IST, Greece: IEEE; 2008.

13. Comité consultatif national d'éthique. Pour les sciences de la vie et de la santé, France: Springer; 2008.

14. G Paxinos, C Watson. The rat brain in stereotaxic systems. USA: Academic Pres; 1998. 